\title{
Um comparativo entre ferramentas utilizadas no ensino baseadas em Cloud Computing
}

\author{
Taciano Balardin de Oliveira - PPGI Universidade Federal de Santa Maria \\ tacianobalardin@gmail.com \\ Érico Marcelo Hoff do Amaral - PPGI Universidade Federal de Santa Maria \\ erico@inf.ufsm.br \\ Roseclea Duarte Medina - PPGI Universidade Federal de Santa Maria \\ roseclea.medina@gmail.com
}

Luis Fabio Faccin - PPGI Universidade Federal de Santa Maria fabiofaccin@gmail.com

\begin{abstract}
Resumo. A crescente utilização da computação em nuvem nos últimos anos proporcionou a criação de novas soluções que impulsionam a comunicação, produtividade e estimulam a colaboração entre acadêmicos e professores de escolas ou universidades. Este artigo tem o objetivo de estudar ferramentas com custos de implementação relativamente baixos, baseadas em Cloud Computing e que podem ser aplicadas nesses ambientes, apresentando comparativos, testes, benefícios e desvantagens de cada uma delas.
\end{abstract}

Palavras-chaves: comparativo, ferramentas, computação em nuvens

\section{A comparative among tools used in education based on Cloud Computing}

\begin{abstract}
The increasing use of Cloud Computing in recent years led to the creation of new solutions that improve communication, productivity and foster collaboration between academics and teachers from schools or universities. This article aims to study tools with relatively low implementation costs, based on cloud computing and can be applied in these environments, presenting comparative, tests, benefits and disadvantages of each.
\end{abstract}

Keywords: comparative, tools, cloud computing

\section{Introdução}

A tecnologia nos traz novas maneiras de pensar, trabalhar, comprar, vender e também de se relacionar. Essas formas se constituem ligeiramente em novos paradigmas, que em alguns casos são tão superiores aos anteriores que às organizações podem não ter outra opção a não ser adotá-los. (Aboulnaga et al, 2009)

Com a evolução tecnológica da sociedade, seja na área educacional, dos negócios ou no âmbito pessoal, as pessoas estão imersas em uma mobilidade na qual o acesso a recursos é necessário a qualquer lugar ou hora, passando a serem consumidores de recursos e pagando por isso de acordo com sua utilização. Para isso, a Internet serve como infraestrutura 
transparente, onde altas quantidades de informações trafegam entre diferentes sistemas e redes. (Kleinrock, 2003)

A Cloud Computing (Computação em Nuvem), de acordo com (Armbrust et al, 2009) é uma forma de se obter infraestrutura e aplicações no modelo pay-as-you-go (pague conforme usar), onde o hardware e o software do centro de processamento de dados são o que chamamos de nuvem e são entregues em forma de aplicações e serviços através da Internet. Os próprios serviços têm sido referidos como Software as a Service (SaaS), Infrastructure as a Service (IaaS), Plataform as a Service (PaaS), entre outros. Mais informações sobre as formas de serviços disponíveis na nuvem podem ser obtidas em (Armbrust et al, 2009) e (Vaquero, 2011).

Contudo, o objetivo deste artigo é estudar as funcionalidades, testar, apontar prós e contras de alguns laboratórios virtuais viabilizados por ambientes baseados em Cloud Computing através do modelo SaaS, que disponibiliza software como serviço para seus usuários.

Este artigo está organizado da seguinte forma: a seção 2 conta com uma descrição do que são laboratórios virtuais e quais foram estudados neste artigo, na seção 3 estão os resultados dos comparativos e testes aplicados a estes ambientes e, por fim, na seção 4 está a conclusão do artigo.

\section{Laboratórios Virtuais em Cloud Computing}

Um laboratório de informática virtual leva programas em execução no hardware da escola ou faculdade para as telas de dispositivos capazes de acessar a Internet, dando aos alunos a possibilidade de criar e salvar trabalhos como se os programas estivessem sendo executados em seus próprios discos rígidos. O desempenho do software depende da velocidade de acesso do aluno à Internet, por isso, mesmo alunos com computadores mais antigos podem dispor desta infraestrutura sem dificuldades.

Em ambientes educacionais, tanto o modelo IaaS quanto o PaaS podem servir como apoio às tecnologias para a educação. Trazendo como beneficio um aumento no acesso aos recursos com redução de custos, ajudando os educadores e alunos a alcançarem os resultados pretendidos de forma mais rápida. (Vaquero, 2011) Além disso, outras empresas tem criado suas próprias ferramentas $\mathrm{SaaS}$ que facilitam o acesso educacional a infraestruturas na nuvem, como por exemplo os serviços que foram estudados neste artigo: Live@Edu da Microsoft, Google Apps for Education da Google e Academic Skills Cloud da IBM.

\subsection{Microsoft: Live@Edu}

O Microsoft Live@Edu é um serviço gratuito que oferece à alunos, professores, pesquisadores e administradores ferramentas fáceis para comunicação, colaboração e compartilhamento de arquivos online que dispensa a instalação de programas específicos para acessá-lo. (Microsoft, 2011) 
Usando um único login e senha com o domínio da instituição, os usuários têm acesso a ferramentas como Outlook Live que é um serviço de e-mail com capacidade para armazenar até $10 \mathrm{~Gb}$ de mensagens, ao serviço de armazenamento de arquivos com até $25 \mathrm{~Gb}$ de espaço denominado Sky Drive, também possuem acesso ao Office Web Apps que são ferramentas semelhantes ao Word, Excel, PowerPoint e OneNote, e ao serviço de mensagens instantâneas Windows Live Messenger que possibilita a conversação em forma de mensagens de texto, voz e vídeo. (Microsoft, 2011) Todos esses recursos podem ser acessados de qualquer lugar com acesso a Internet, seja no computador de casa, da instituição ou através de um dispositivo móvel.

O Live@Edu também reforça o vínculo dos estudantes com seu estabelecimento educacional, pois pode ser personalizado com o logo da instituição. Além disso, possui ferramentas administrativas que geram diversos relatórios de uso do domínio, das ferramentas e tendências de uso dos serviços. (Microsoft, 2011)

\subsection{Google: Google Apps for Education}

O Google Apps for Education é um serviço gratuito do Google que, assim como o serviço da Microsoft, tem a proposta de aperfeiçoar a comunicação, compartilhamento e colaboração entre as pessoas de forma rápida, potencializando a interação entre os mesmos e sem precisar de instalação de programa específico para utilizá-lo. (Google, 2011)

Os serviços disponíveis no Google Apps for Education são compostos por uma ferramenta de e-mail com capacidade para armazenar até $7 \mathrm{~Gb}$ de dados, que é integrada a um serviço de mensagens instantâneas, de videoconferência e com um serviço de agenda capaz de gerenciar atividades de forma colaborativa, também possui uma ferramenta para edição e compartilhamento de textos, planilhas, apresentações e questionários, além disso, possibilita a criação de sites para o compartilhamento de notícias, vídeos e outras informações em grupo. (Google, 2011) Assim como no Live@Edu, todas estas ferramentas estão disponíveis para acesso através da Internet, tanto do computador de casa quanto no da instituição ou também através de dispositivos móveis.

O Google Apps for Education pode ser personalizado de acordo com a instituição e possui ferramentas administrativas para gerenciar usuários e personalizar todos os serviços disponíveis. (Google, 2011)

\subsection{IBM: Academic Skills Cloud}

Lançado em 2010, o Academic Skills Cloud inicialmente foi disponibilizado apenas para 20 universidades americanas, atualmente a iniciativa está aberta para membros do corpo docente em mais de 4.500 universidades no mundo. Os usuários desta ferramenta são capazes de acessar softwares da IBM em salas de aulas e laboratórios sem ser necessário instalar os produtos em seus próprios sistemas. (IBM, 2010)

A ferramenta disponibiliza aos docentes de forma gratuita mais de 20 imagens de máquinas virtuais contendo produtos da IBM que podem ser executados na nuvem, tais como o IBM Rational, WebSphere, Lotus, Gestão da Informação, Cognos e software Tivoli. A empresa ainda planeja adicionar outros softwares a nuvem. (IBM, 2010) 
4

Para acessar as ferramentas disponibilizadas pela IBM, o docente deve preencher o formulário disponível no site da Academic Skills Cloud solicitando acesso ao ambiente. Os dados enviados serão analisados pela equipe da IBM e se validados o usuário receberá acesso ao serviço de forma gratuita.

\subsection{Utilização Para Fins Pedagógicos}

Em consonância com (Convergência Digital, 2011), em Agosto de 2011 a escola primária $\mathrm{n}^{\mathrm{o}}$ 34, da cidade de Tigre, na Argentina, tornou-se a primeira da América Latina a utilizar a nuvem pública do Google com fins pedagógicos. Nos próximos quatro anos a ideia é que as 56 escolas existentes na cidade de Tigre estejam integradas, disponibilizando o serviço a 35 mil alunos.

Já no Brasil, a Universidade Luterana do Brasil utiliza o serviço Live@Edu da Microsoft, de acordo com (Neves, 2011), no segundo semestre de 2011 devem ser ativadas cerca de 100 mil contas que incluem perfis variados de usuários (alunos e professores). Além disso, a Universidade estima que tenha economizado em torno de R 2 milhões com a adoção deste serviço.

\section{Comparativos e Testes}

A crescente utilização de dispositivos móveis, como celulares e tablets, aliado, em alguns casos, à baixa velocidade de conexão de dados, tornam a velocidade de carregamento de serviços online um ponto importante a ser avaliado, onde usuários podem até ser forçados a buscar um serviço semelhante que possa suprir suas necessidades caso o desempenho do mesmo não seja satisfatório. $\mathrm{O}$ primeiro teste realizado define o tempo de carregamento das páginas que compõem cada recurso. Uma página muito lenta poderá demorar muito tempo para ser carregada, podendo causar aborrecimento por parte do usuário que provavelmente desistirá de acessar o serviço.

Para medir a velocidade de carregamento das páginas, foi utilizada a ferramenta Firebug v1.7.2. O Firebug (Firebug, 2011) está disponível gratuitamente em formato de plugin para o Mozilla Firefox e dispõe de diversas ferramentas, como monitor de HTML, CSS, JavaScript, dentre outras, como tempo de carregamento dos objetos que compõem a página. Estas ferramentas geralmente são utilizadas por desenvolvedores para identificar e eliminar erros de programação e edição das páginas web.

O plugin mostra uma lista com todos os objetos que compõem a página, seus tamanhos e o tempo que foi necessário para o carregamento de cada um deles. A diferença entre o tempo inicial do primeiro objeto e o tempo final do último objeto caracteriza o tempo total de carregamento da página.

Um segundo teste pôde ser aplicado utilizando a ferramenta Web Developer v1.1.9, também disponível gratuitamente em forma de plugin para o Mozilla Firefox. (Web Developer, 2011) Esta ferramenta dispõe de recursos mais específicos no tratamento de erros durante o desenvolvimento, como redimensionamento de janelas, depuração de formulários e 
validação de links. Um dos pontos mais interessantes abordados por esta ferramenta é a capacidade de analisar os seguintes aspectos:

Conformidade com padrões web: um padrão web pode ser definido como um conjunto de normas e recomendações de caráter técnico, produzidos pelo W3C (World Wide Web Consortium) e destinados a orientar os desenvolvedores para a criação de páginas que possam ser acessadas independentemente dos dispositivos usados ou de suas necessidades especiais (Silva, 2011). Uma página que não esteja em conformidade com as regras de acessibilidade definidas pelos padrões web poderá ter seu acesso prejudicado em dispositivos móveis ou em leitores de telas utilizados por deficientes visuais.

Erros de CSS: as folhas de estilo (Cascading Style Sheets) são documentos escritos em uma linguagem de marcação específica capazes de definir o formato das páginas $w e b$. Sua principal função é separar o layout do conteúdo das páginas e facilitar o acesso a estas páginas em qualquer dispositivo, utilizando os padrões web.

Erros de JavaScript: o JavaScript permite a programação e utilização de pequenos scripts nas páginas. Estes pequenos programas podem alterar a página dinamicamente, realizar a validação de formulários, modificar recursos visuais e realizar cálculos. Erros de JavaScript podem interromper ou gerar alertas durante o carregamento e execução das páginas.

Os demais testes baseiam-se na comparação dos diferentes serviços oferecidos pelos laboratórios virtuais, bem como os recursos que cada um deles disponibiliza. O serviço da IBM não pode ser testado devido ao fato de que ele está disponível apenas para docentes e até então não foi liberado acesso ao ambiente.

\subsection{Velocidade de Acesso}

Para medir a velocidade de acesso em cada serviço, foram realizadas três requisições seqüenciais, a partir dos dados obtidos foram calculadas as médias do tempo de acesso. Os testes foram realizados com a utilização do plugin Firebug (Figura 1) e por meio de três velocidades de conexões, sendo elas duas conexões de banda larga com velocidades de $1 \mathrm{Mbps}$ e $1,5 \mathrm{Mbps}$ e uma conexão discada com velocidade de $56 \mathrm{Kbps}$.

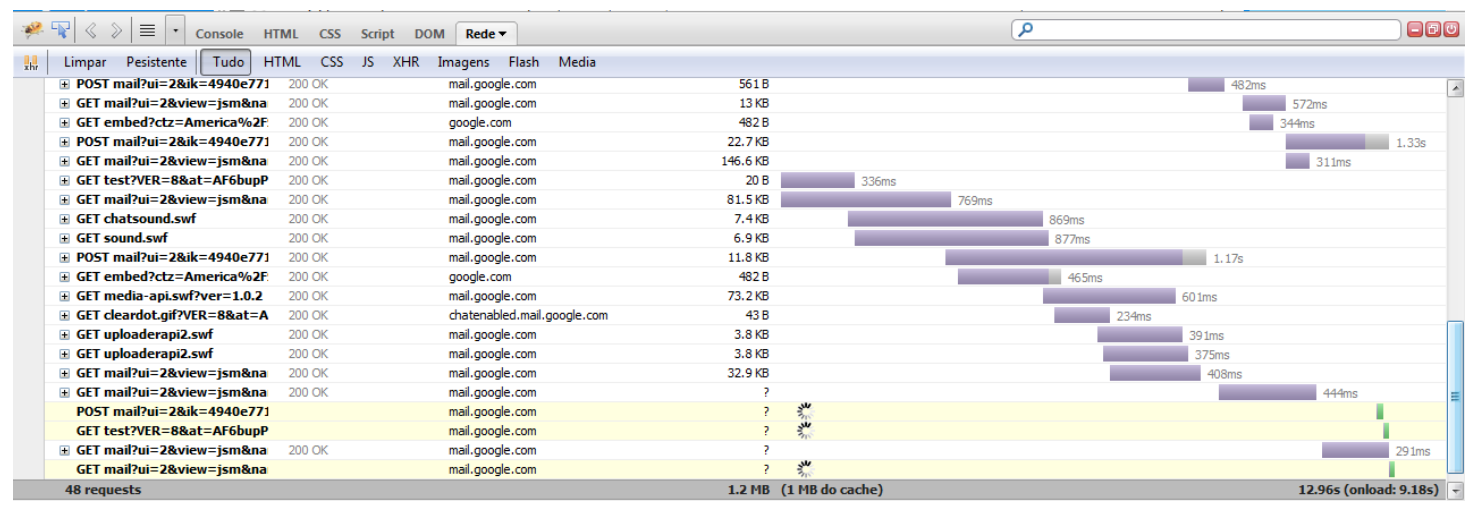

Figura 1 - Plugin Firebug em execução. 


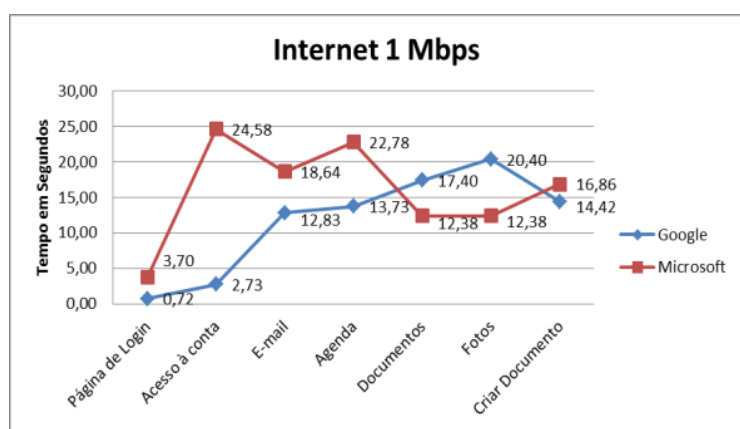

Figura 2 - Comparativo entre serviços utilizando Internet de $1 \mathrm{Mbps}$.

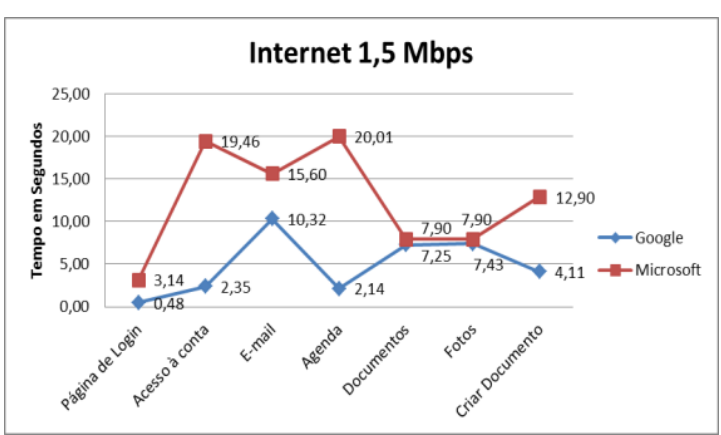

Figura 3 - Comparativo entre serviços utilizando Internet de 1,5Mbps.

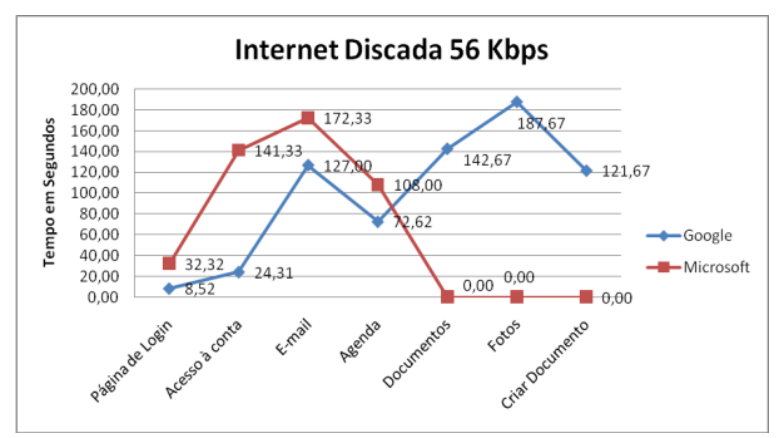

Figura 4 - Comparativo entre serviços utilizando Internet de 56Kbps.

Conforme o comparativo entre os serviços oferecidos pelas duas empresas expostos na Figura 2, Figura 3 e Figura 4, pode se perceber que a maioria dos serviços proporcionados pelo Google possui um tempo de acesso menor que os oferecidos por sua concorrente. Entretanto, nos testes realizados com internet de $1 \mathrm{Mbps}$, os serviços de documentos e fotos do Google tiveram um tempo de carregamento superior, fato que curiosamente não ocorreu durante os testes utilizando Internet de 1,5Mbps. Já utilizando a Internet com conexão discada os tempos de carregamento foram muito superiores em relação aos testes com banda larga, além disso, três dos testes que foram realizados com os serviços da Microsoft nesta mesma conexão discada, mesmo após uma espera de mais de 5 minutos o tempo de carregamento não pode ser definido, pois os serviços não foram carregados com sucesso (Documentos, Fotos e Criar Documento).

Também através dos testes com o Firebug, foi possível avaliar que as páginas da empresa Microsoft possuem um número maior de objetos à serem carregados em relação às páginas do Google, o que torna a tarefa do carregamento das páginas mais demorada.

\subsection{Erros e conformidade com padrões web}

O segundo teste apresenta uma abordagem voltada à questão do tratamento de erros de CSS, JavaScript e da acessibilidade. Na Tabela 1 é apresentado o resultado dos testes, onde " $\mathrm{x}$ " significa que possui algum erro ou não é compatível com os padrões e " $\mathrm{v}$ " tratam-se dos itens onde não existem erros ou são compatíveis com os padrões $w e b$.

Tabela 1 - Teste de erros e conformidade com padrões web.

\begin{tabular}{|c|c|c|}
\hline Google & Microsoft \\
\cline { 2 - 3 }
\end{tabular}




\section{7}

\begin{tabular}{|l|c|c|c|c|c|c|} 
& $\begin{array}{c}\text { Padrões } \\
\text { Web }\end{array}$ & $\begin{array}{c}\text { Erros de } \\
\text { CSS }\end{array}$ & $\begin{array}{c}\text { Erros de } \\
\text { JavaScript }\end{array}$ & $\begin{array}{c}\text { Padrões } \\
\text { Web }\end{array}$ & $\begin{array}{c}\text { Erros de } \\
\text { CSS }\end{array}$ & $\begin{array}{c}\text { Erros de } \\
\text { JavaScript }\end{array}$ \\
\hline Página de login & $\mathrm{x}$ & $\mathrm{v}$ & $\mathrm{v}$ & $\mathrm{x}$ & $\mathrm{x}$ & $\mathrm{v}$ \\
\hline Acesso à conta & $\mathrm{x}$ & $\mathrm{x}$ & $\mathrm{v}$ & $\mathrm{x}$ & $\mathrm{x}$ & $\mathrm{v}$ \\
\hline E-mail & $\mathrm{v}$ & $\mathrm{x}$ & $\mathrm{v}$ & $\mathrm{v}$ & $\mathrm{x}$ & $\mathrm{v}$ \\
\hline Agenda & $\mathrm{v}$ & $\mathrm{x}$ & $\mathrm{v}$ & $\mathrm{v}$ & $\mathrm{x}$ & $\mathrm{v}$ \\
\hline Documentos & $\mathrm{v}$ & $\mathrm{x}$ & $\mathrm{v}$ & $\mathrm{v}$ & $\mathrm{x}$ & $\mathrm{v}$ \\
\hline Fotos & $\mathrm{v}$ & $\mathrm{x}$ & $\mathrm{v}$ & $\mathrm{v}$ & $\mathrm{x}$ & $\mathrm{v}$ \\
\hline Criar documento & $\mathrm{v}$ & $\mathrm{x}$ & $\mathrm{v}$ & $\mathrm{v}$ & $\mathrm{x}$ & $\mathrm{v}$ \\
\hline
\end{tabular}

Os resultados obtidos foram muito semelhantes e todos os serviços de ambas as empresas apresentam pelo menos um tipo de problema. O principal problema que afeta todos os serviços da Microsoft e a maioria dos serviços do Google são erros de CSS. Estes erros variam entre uma propriedade descrita de forma errônea (ex: cores, fontes) até propriedades que não sejam compatíveis ou interpretadas por todos os navegadores. $\mathrm{O}$ segundo tipo de erro que afeta os serviços de login e acesso à conta é a inconformidade com os padrões web, em específico a maneira como o browser interpreta os arquivos CSS. Esta inconformidade pode ocasionar falhas no acesso quanto for utilizado dispositivo móvel ou através de sistemas leitores de telas.

Um caso especial pode ser percebido por não haver erros de JavaScript, ou seja, as chances de se obter uma falha no carregamento e utilização das páginas são praticamente nulas, a menos que haja algum problema no interpretador de JavaScript do navegador, uma falha na conexão, ou um erro de lógica no próprio código.

\subsection{Comparativos entre as ferramentas}

Além dos testes de velocidade de acesso, de erros relativos à CSS ou JavaScript e da conformidade com padrões de acessibilidade, também foram elaboradas tabelas comparativas dos serviços prestados pela Microsoft e pelo Google de um modo geral, como apresentado na Tabela 2.

Tabela 2 - Comparativo geral entre os serviços.

\begin{tabular}{|c|c|c|}
\hline Características & Google & Micros oft \\
\hline Permissão de uso & $\begin{array}{l}\text { - Necessário ter domínio web próprio; } \\
\text { - Preencher formulário de cadastro. }\end{array}$ & $\begin{array}{l}\text { - Necessário ter domínio web próprio; } \\
\text { - Preencher formulário de cadastro. }\end{array}$ \\
\hline $\begin{array}{l}\text { Requisitos } \\
\text { mínimos }\end{array}$ & $\begin{array}{l}\text { - Necessário apenas computador com } \\
\text { acesso à internet. }\end{array}$ & $\begin{array}{l}\text { - Necessário apenas computador com } \\
\text { acesso à internet. }\end{array}$ \\
\hline $\begin{array}{l}\text { Recursos } \\
\text { disponíveis }\end{array}$ & $\begin{array}{l}\text { - E-mail, Aplicativos, Agenda, Álbum de } \\
\text { Fotos, Documentos, Mapas, Leitor de } \\
\text { RSS, Estatísticas de acesso à páginas da } \\
\text { internet, Notas, Mensageiro Instantâneo, } \\
\text { Criação de Sites. }\end{array}$ & $\begin{array}{l}\text { - E-mail, Agenda, Álbum de Fotos, } \\
\text { Documentos, Armazenamento, } \\
\text { Mensageiro Instantâneo. }\end{array}$ \\
\hline $\begin{array}{l}\text { Vantagens em } \\
\text { comum da } \\
\text { interface como } \\
\text { usuário }\end{array}$ & \multicolumn{2}{|c|}{$\begin{array}{l}\text { - Permite customização de acordo coma instituição ou empresa; } \\
\text { - Permite a utilização de e-mail personalizado; } \\
\text { - Utilização de perfis para cada usuário; } \\
\text { - Possuem relatórios gerenciais; } \\
\text { - Visualização de utilização dos recursos; }\end{array}$} \\
\hline
\end{tabular}




\begin{tabular}{|c|c|c|}
\hline & \multicolumn{2}{|c|}{$\begin{array}{l}\text { - Permitem compartilhamento de arquivos em tempo real; } \\
\text { - As permis sões de acesso, utilização e edição de conteúdo ou serviços podem ser } \\
\text { personalizadas de modo individual. }\end{array}$} \\
\hline $\begin{array}{l}\text { Vantagens } \\
\text { Individuais }\end{array}$ & $\begin{array}{l}\text { - Visual limpo; } \\
\text { - Tempo de carregamento menor; } \\
\text { - Mais recursos; } \\
\text { - Possui software para gerenciamento e } \\
\text { upload de imagens. }\end{array}$ & $\begin{array}{l}\text { - Serviços integrados (fotos, arquivos, } \\
\text { documentos); } \\
\text { - Melhor gerenciamento de arquivos (por } \\
\text { ser integrado); } \\
\text { - Maior espaço de armazenamento; } \\
\text { - Editor de documentos semelhante ao } \\
\text { Pacote Office. }\end{array}$ \\
\hline $\begin{array}{l}\text { Desvantagens } \\
\text { Individuais }\end{array}$ & $\begin{array}{l}\text { - Não possui um serviço dedicado } \\
\text { exclusivamente para armazenamento } \\
\text { - Espaço de armazenamento menor que } \\
\text { ao disponibilizado pelo concorrente. }\end{array}$ & $\begin{array}{l}\text { - Nos testes realizados as páginas } \\
\text { demoraram mais para carregar em relação } \\
\text { ao concorrente. }\end{array}$ \\
\hline
\end{tabular}

A Tabela 3 mostra uma comparação entre cada um dos serviços em comum, disponibilizados pelo Google e pela Microsoft.

Tabela 3 - Comparativo geral entre os serviços, por ferramentas.

\begin{tabular}{|c|c|c|}
\hline & Google & Micros oft \\
\hline $\begin{array}{l}\text { Serviço de } \\
\text { E-mail }\end{array}$ & $\begin{array}{l}\text { - Arquivamento em tags; } \\
\text { - Agrupamento de conversas; } \\
\text { - Espaço para armazenamento de } 7 \mathrm{~Gb} \text {; } \\
\text { - Mensageiro instantâneo integrado; } \\
\text { - Possui complementos: "Google Labs". }\end{array}$ & $\begin{array}{l}\text { - Arquivamento em pastas; } \\
\text { - Não agrupa conversas; } \\
\text { - Espaço para armazenamento de } 10 \mathrm{~Gb} \text {; } \\
\text { - Possui mensageiro instantâneo. }\end{array}$ \\
\hline $\begin{array}{l}\text { Serviço de } \\
\text { Documentos }\end{array}$ & $\begin{array}{l}\text { - Interface básica; } \\
\text { - Edição simultânea para vários usuários; } \\
\text { - Limite inicial } 1 \mathrm{~Gb} \\
\text { - Possui serviço de questionários. }\end{array}$ & $\begin{array}{l}\text { - Interface semelhante ao pacote Office; } \\
\text { - Não permite edição simultânea; } \\
\text { - Serviço integrado para armazenamento } \\
\text { de arquivos e imagens; } \\
\text { - Limite compartilhado entre todos os } \\
\text { armazenamentos com } 25 \mathrm{~Gb} \text {. }\end{array}$ \\
\hline $\begin{array}{l}\text { Serviço de } \\
\text { Agenda }\end{array}$ & \multicolumn{2}{|c|}{$\begin{array}{l}\text { - Ambos apresentam visualização de compromis sos mensal, semanal, diário; } \\
\text { - Compartilhamento de compromissos; } \\
\text { - Lista de tarefas; } \\
\text { - Diferencial: O Google envia mensagens por SMS alertando os compromissos. }\end{array}$} \\
\hline $\begin{array}{l}\text { Serviço de } \\
\text { Armazena- } \\
\text { mento }\end{array}$ & - Serviço compartilhado com documentos. & - Serviço compartilhado com documentos. \\
\hline $\begin{array}{l}\text { Serviço de } \\
\text { Imagens }\end{array}$ & $\begin{array}{l}\text { - Possui Serviço dedicado; } \\
\text { - Capacidade para armazenar } 1 \mathrm{~Gb} ; \\
\text { - Possui software para gerenciar; } \\
\text { - Permite o compartilhamento de álbuns, } \\
\text { tags, reconhecimento facial e localização } \\
\text { em mapas. }\end{array}$ & - Serviço compartilhado com documentos. \\
\hline
\end{tabular}

\section{Conclusão}

As ferramentas elaboradas pela Microsoft, Google e IBM que foram estudadas neste artigo possibilitam que alunos e professores tenham acesso a uma tecnologia que impulsiona a produtividade, comunicação e principalmente estimula a colaboração, sendo que nos serviços da Microsoft e Google isso pode ser percebido através dos testes e estudos realizados, já no da IBM pode-se destacar tais características pelo material teórico disponibilizado no site da ferramenta. 
9

Por funcionarem na nuvem, as instituições que adotam tais tecnologias não se preocupam com a complexidade de manutenção de hardware e software para manter o ambiente funcional e disponível. Além disso, soluções de ensino baseadas em computação na nuvem são benéficas, pois nem sempre as escolas e faculdades dispõem de recursos para implementarem sistemas virtuais que auxiliem os alunos em seu aprendizado.

Entretanto, assim como em diversas outras aplicações que funcionam na nuvem, existe ainda um grande receio por parte das instituições de ensino quanto à segurança das informações que estão armazenas nesses ambientes, fazendo com que nos sites dos desenvolvedores das aplicações esta seja uma questão tratada de forma destacada e bem explicada, na tentativa de conscientizar os utilizadores quanto à segurança de tais ambientes.

Com a análise dos testes e comparativos podemos concluir que a solução apresentada pelo Google possui algumas vantagens em relação à da Microsoft, uma vez que suas páginas podem ser visualizadas com maior velocidade e seus serviços apresentam características importantes, como compartilhamento de arquivos e edição simultânea. Além disso, alguns serviços oferecidos pela ferramenta da Microsoft não carregaram durante os testes realizados utilizando conexão discada. $\mathrm{O}$ fato da Google possuir um menor espaço para armazenamento não chega a ser um fator limitante, já que é possível adquirir maior capacidade de armazenamento com um custo relativamente reduzido (centavos de dólar anuais para cada gigabyte adicional).

\section{Referências Bibliográficas}

ABOUlNAGA A., SALEM K., SOROR A., MINHAS U., KOKOSIELIS P., KAMATH S. (2009). Deploying Database Appliances in the Cloud. IEEE Data Eng. Bull., Vol. 32, No. 1., pp. 13-20.

ARMBRUST M., FOX A., GRITH R., JOSEPH A., KATZ R., KONWINSKI A., LEE G., PATTERSON D., RABKIN A., STOICA I., ZAHARIA M. (2009). Above the clouds: A berkeley view of cloud computing. Technical Report UCB/EECS-2009-28, EECS Department, University of California, Berkeley, Feb 2009.

CONVERGÊNCIA DIGITAL - Hotsite Cloud Computing (2011). Escolas Argentinas

Começam a Usar Cloud Computing. Disponível em:
$<\mathrm{http} / /$ convergenciadigital.uol.com.br/cgi/cgilua.exe/sys/start.htm?infoid=27494\&sid=97>. Acesso em Novembro de 2011.

FIREBUG. Add-ons para Mozilla Firefox. Disponível em: $<$ https://addons.mozilla.org/ptbr/firefox/addon/firebug/>. Acesso em Junho de 2011.

GOOGLE. Google Apps for Education (2011). Disponível em: $<$ http:/www.google.com/apps/int/en/edu/>. Acesso em Junho de 2011.

IBM. IBM Academic Skills Cloud (2010). Disponível em: $<\mathrm{http} / /$ www.ibm.com/developerworks/university/cloud/asc_index.html $>$. Acesso em Junho de 2011. 
10

KLEINROCK L. (2003). An Internet vision: the invisible global infrastructure. Ad Hoc Networks, Vol. 1, No. 1., pp. 3-11. doi:10.1016/S1570-8705(03)00012-X

MICROSOFT. Live@Edu (2011). Disponível em: <http://www.microsoft.com/liveatedu/>. Acesso em Junho de 2011.

NEVES, G. (2011). Ulbra Economiza R\$ 2 milhões com Live@Edu. Disponível em: $<$ www.baguete.com.br/noticias/software/04/05/2011/ulbra-economiza-r-2-mi-com-liveedu>. Acesso em Novembro de 2011.

SILVA, M. S. O que é CSS? E Web Standards? Disponível em: $<$ http $/ /$ www.maujor.com/index.php $>$. Acesso em Junho de 2011.

VAQUERO, L. M. (2011) EduCloud: PaaS versus IaaS Cloud Usage for an Advanced Computer Science Course. IEEE Education Society, Vol 54 No. 4 p. 590-598.

WEB DEVELOPER (2011). Add-ons para Mozilla Firefox. Disponível em: $<$ https://addons.mozilla.org/en-US/firefox/addon/web-developer/>. Acesso em Junho de 2011. 